\title{
The Invisible Thin Red Line
}

\author{
Giuliano Torrengo \& Samuele Iaquinto \\ Penultimate version, to appear in Pacific Philosophical Quarterly
}

\begin{abstract}
The aim of this paper is to argue that the adoption of an unrestricted principle of bivalence is compatible with a metaphysics that (i) denies that the future is real, (ii) adopts nomological indeterminism, and (iii) exploits a branching structure to provide a semantics for future contingent claims. To this end, we elaborate what we call Flow Fragmentalism, a view inspired by Kit Fine (2005)'s non-standard tense realism, according to which reality is divided up into maximally coherent collections of tensed facts. In this way, we show how to reconcile a genuinely A-theoretic branching-time model with the idea that there is a branch corresponding to the thin red line, that is, the branch that will turn out to be the actual future history of the world.
\end{abstract}

\section{Keywords}

Thin Red Line, Presentism, Fragmentalism, Branching Time, Principle of Bivalence

\section{Presentism and Bivalence Failure}

Presentists hold that the present constitutes the whole of reality. Given that at present the future is not yet here, future contingent claims, such as: 'There will be a sea battle tomorrow', lack grounds - i.e., there is no fact of the matter to their truth or falsity. A presentist who agrees with this line of thought, and maintains that any truth requires a ground 1 may be tempted to think that future contingent claims are neither true nor false, and thus to deny that

\footnotetext{
${ }^{1}$ That is, either something exists that makes it true, or it is made true by the fact that certain patterns of properties and relations are instantiated. Although we will often use "fact" talk in what follows, we are not assuming the existence of facts as a distinct ontological category - as it will be clear when we will introduce our official idiom.
} 
the principle of bivalence holds unrestrictedly $\mathrm{L}^{2}$ Bivalence, though, is a very elegant and wellbehaved logical property, which we should not lightly dismiss on the ground of our preferred temporal metaphysics. Is there any strategy that the presentist can endorse to save bivalence? Two options present themselves quite naturally.

The first option is to endorse nomological determinism. If present truths, together with the laws of nature, necessitate future tensed truths, there is no reason to deny a determined truthvalue to future contingent claims even if they lack future grounds (see Markosian 2013). But the issue of nomological determinism vs. nomological indeterminism is an empirical one, and it would be nice if the presentist could save bivalence even if the universe we happen to live in turns out to have indeterminist laws. Therefore, in what follows, we will assume that nomological indeterminism holds.

The second option is to introduce in the present reality "brute facts" about which history among all the nomologically possible alternatives will be the actual one. If we represent, as is customary, nomologically possible histories through a tree-like structure, such brute facts would single out among all future branches a thin red line - i.e. the branch that will turn out to be the actual future history of the world $\mathrm{S}^{3}$

We will not discuss here the viability or the costs of this strategy for presentism, understood as a standard form of tense realism (in Kit Fine 2005's sense) 4 Rather, we will explore a non-standard form of tense realism, which we label "Flow Fragmentalism"

\footnotetext{
2 See, for instance, Bourne (2006). In a similar vein, Le Poidevin (1991: 38) observes that "the extent to which the principle of bivalence is violated by statements about the past or future depends, for [the presentist], upon how much causal determinism he is prepared to allow. [...] In an indeterministic universe [...] many statements about the future must [...] lack a truth-value". To some extent, such a position might be thought of as sympathetic to Markosian (2013: 137)'s one. The latter seems to think that within a presentist framework, if laws of nature turned out to be wildly indeterministic, it would be impossible to evaluate contingent truths about the past as true or false. And, we add, similar considerations could be easily applied to future contingent claims.

3 See Prior (1967: Chap. 7) and Øhrstrøm (2009). Linear time is compatible with indeterminism, but it is usually argued that to vindicate the intuition that the future is open we need branching and bivalence failure (see Belnap et al 2001; see Barnes and Cameron 2009 for a criticism of the idea and Torre 2011 for an overview). Here, we are not interested in whether branching or branching together with a thin red line vindicate any alleged intuition about the openness of the future. Our aim is to show that bivalence and a certain way to understand the unreality of the future are compatible even on the assumption of nomological indeterminism and branching time.

4 See Borghini and Torrengo (2012) for a discussion of some of the disadvantages.

5 We are here elaborating and expanding on the theory set forth in Torrengo and Iaquinto (2019), and Iaquinto (2019).
} 
endorsing it we can avert the failure of bivalence by introducing a thin red line, while denying the reality of the future and buying nomological indeterminism.

Both presentists and flow fragmentalists maintain that the future is unreal. What does the presentist mean by such a claim? We can make it more precise by describing reality as constituted by a variety of tensed facts. Consider the tensed fact that Socrates is sitting (now) - as opposed to the tenseless fact that Socrates is sitting at $t$. If Socrates is presently sitting, reality is constituted by the fact that Socrates is sitting (now). Assume that in a few minutes Socrates will be standing: can the presentist accept the future fact that corresponds to such a future-tensed truth? It depends on how we read 'future fact'. A future fact in a weak sense is a future-tensed fact that obtains at present. If Socrates will be standing in a few minutes, the fact that Socrates will be standing obtains at present (and hence it constitutes reality now). A future fact in a strong sense is a present-tensed fact that will obtain in the future. If Socrates will be standing in a few minutes (and he is sitting now), the fact that Socrates is standing will obtain in a few minutes (and hence it will constitute reality, which it does not now).

Presentists can accept future facts in the weak sense, at least insofar as they resort to slightly "exotic" but presentism-friendly ideology or ontology - for instance, by resorting to "Lucretian" properties. The Lucretian presentist takes properties such as "being such that Socrates will be standing" to be an irreducible element of reality, and identify the future fact, in the weak sense, that Socrates will be standing with the fact that the mereological sum of all the presently existing things is such that Socrates will be standing 6 However, presentism is not compatible with accepting future facts in the strong sense. Indeed, for the presentist future facts in the strong sense are not facts at all, i.e., the extension of the very concept is empty. The facts that obtain at present are the facts that obtain simpliciter, and that constitute reality, namely the only facts there are. Thus, according to presentism, the future is unreal in the sense that there are no future facts in the strong sense.

Flow Fragmentalism is the thesis that the temporal dimension is constituted by internally coherent pluralities of tensed facts (fragments) that are mutually incompatible. This means that although absolutely speaking reality is constituted by future facts in the strong sense (since each

6 See Bigelow (1996). Other options in the literature are adopting primitively tensed relations (Brogaard 2006 and Brogaard 2013), or to adopt the haecceitist version of presentism (Keller 2004, Ingram 2016a, 2016b), which defines past and future entities as uninstantiated "thisnesses". Still another option is to embrace a form of ersatzer presentism. The ersatzer takes times to be maximal abstract objects, that is, abstract representations of a given state of the world (see Bourne 2006 and Crisp 2007). 
fragment constitutes reality on a par with all the others), within each fragment only future facts in the weak sense obtain, and thus the future is unreal in a sense utterly analogous to the presentist one. In other terms, it is possible to take seriously both the idea that the future is nothing over and above the plethora of nomological alternatives that the laws of nature presently allow, and that reality is constituted by a "fragmented", albeit genuinely dynamic, temporal dimension.

The main thesis of this paper is that we can build a fiction about a tree-like structure of series of fragments that mimics the nomological alternatives that we find within each fragment. Given that only one of the "histories" of fragments can be taken to be part of reality, the fiction will contain a thin red line (the series of fragments that correspond to the ones that constitute reality), although an "invisible" one, since it is neither grounded on the facts that obtain within any fragment, nor can be retrieved from an all-encompassing über-perspective. Within a framework of this kind, one can naturally adopt unrestricted bivalence for future contingents without embarking further costs. Let us then turn to Flow Fragmentalism.

\section{Absolute Constitution and Relative Obtainment}

Flow Fragmentalism combines the intuition that the present is in some sense privileged, with the seeming opposed one that there is no substantive difference among distinct time:7. Standard tense realism (StTR) maintains that tensed facts, such as the fact that Socrates is sitting (now), constitute a coherent reality in an absolute sense. More precisely, StTR is the conjunction of the following three claims (Fine 2005: 270-2) 8

Realism Reality is constituted (at least, in part) by tensed facts.

Absolutism The constitution of reality is an absolute matter, i.e. not relative to a time or other form of temporal standpoint.

Coherence Reality is not contradictory; it is not constituted by facts with incompatible content 9

Note that - for reasons that we do not need to consider here but briefly in the next footnote - StTR proves to be incompatible with the claim that facts obtaining at any time constitute

\footnotetext{
${ }^{7}$ See Merlo (2013), who talks about the specialness and the egalitarian intuitions.

8 See also Fine (2006: 399-400).

${ }^{9}$ As Correia \& Rosenkranz (2011) rightly stress, Fine takes coherence as a primitive notion, which is distinct and more specific than the ordinary notion. If there is no time in which both Socrates is furious and Plato is anxious, then those two facts do not cohere, in Fine's sense, although the two propositions that state those facts form a coherent (in the usual sense) set. See also note 11.
} 
reality in the same way; that is, the following thesis:

Neutrality No time is privileged; the tensed facts that constitute reality are not oriented towards one time as opposed to another 10

Non-standard forms of tense realism maintain Neutrality, while giving up either Absolutism or Coherence. Those who reject Absolutism embrace what Fine (2005) calls External Relativism. External relativists think of reality as a plethora of perspectives, each centred on a time, and the constitution of reality by tensed facts as irreducibly relative. The crucial point is that incompatible tensed facts constitute reality only relative to different perspectives, and there is no overall perspective encompassing all of them (it would be incoherent).

Those who reject Coherence, while keeping Absolutism, adopt Fragmentalism. The fragmentalist drops the assumption that reality is "of a whole", by thinking of reality as constituted by incompatible tensed facts. More precisely, reality is divided up into maximally coherent collections of tensed facts, called fragments. Each fragment is internally coherent, but the whole of reality is not. That reality is incoherent does not entail that conjunctions of incompatible facts can obtain. Suppose for example that Socrates is now sitting and then standing. In describing such a case, fragmentalism resorts to two different fragments, one in which we find all past-, present- and future-tensed facts that obtain when the fact that Socrates is sitting obtains, and another in which we find all past-, present- and future-tensed facts that obtain when the fact that Socrates is standing obtains. Both the fact that Socrates is sitting (now) and the fact that Socrates is standing (now) constitute reality in an absolute sense, but the fact that Socrates is standing (now) obtains in a fragment of reality different from the one in which the fact that Socrates is sitting (now) obtains, and there is no fragment where their conjunction holds. Hence, even though constitution of reality is not irreducibly relative, facts obtain only relative to fragments.

While for the standard tense realist obtainment in the present is obtainment simpliciter, for the fragmentalist there is no obtainment simpliciter, but only within a fragment. Yet, constitution is absolute (contrary to what the external relativist maintains), and so past and future facts in

10 As Fine (2005: 272) points out - when coupled with Absolutism, Coherence, and Neutrality - Realism gives rise to a version of McTaggart (1908)'s Paradox. If some qualitative variation through time occurs, reality will be constituted by incompatible tensed facts. Suppose for example that at $t$ Socrates is sitting, while at $t_{1}$ he is standing. In the light of Realism, at $t$ the tensed fact that Socrates is sitting (now) obtains, while at $t_{1}$ the tensed fact that Socrates is standing (now) obtains. Assuming both Neutrality and Absolutism, those two incompatible facts will constitute reality absolutely speaking and not with respect to a given time. But, under the hypothesis that Coherence holds, reality cannot contain incompatible facts. 
the strong sense are facts, since they constitute reality, even if they do not obtain at present ${ }^{11}$ Thus, in our take on the position, fragmentalism requires a form of double talk: the absolute talk of constitution and the irreducibly relative talk of obtainment. It is important to stress that the latter does not represent merely a "coherence" constraint on how we can describe the reality constituted by incoherent facts. Each way of talking is metaphysically fundamental: as a whole, reality is incoherent (after all, Coherence does not hold), even though there is no instance of a conjunction of incoherent facts, since along the temporal dimension reality is coherently fragmented - viz. divided into fragments constituted by facts that cohere with one another 12

Bearing these remarks in mind, we will now formulate Flow fragmentalism in its official idiom, which does not require commitment to existence of facts as a distinct ontological category 13 We begin with a propositional tensed language $L^{0}$ containing simple present tense sentences $p_{1}, p_{2}, \ldots$, two tense operators $W A S$ and $W I L L$, and two connectives $\neg$ and $\wedge$. The atomic sentences of $L^{0}$ can be thought of as expressing the facts that constitute reality. By resorting to $L^{0}$, in the official idiom, rather than stating that the fact that $p$ constitutes reality, we simply

11 We are aware that this is not the only way to cash out Fine's idea. Lipman (2015), for instance, offers a different interpretation of the view, by characterising it through the elucidation of a primitive notion of coobtainment, rather than by distinguishing relative obtainment from absolute constitution. Roughly, when two facts co-obtain "they form a unified qualitative manifestation of the relevant objects, one single bit of world within which the things are a certain way" (p. 3127), and when two facts do not co-obtain "relative to the one fact, the other fact is not there at all" (p. 3128). Those remarks would suit us as glosses to our notions of "obtaining within the same fragment" and "obtaining in two different fragments" (respectively). Lipman also stresses that "fragmentalism is not the view that there literally speaking are entities called fragments relative to which thing obtain" (p. 3129). We agree with this last remark, since - as we will stress below - (i) we do not allow quantification over fragments, and (ii) relativisation of obtainment to fragment needs to be coupled with absolute constitution to give us fragmentalism. A full discussion of differences and similarity between Lipman's proposal and the view exposed here is beyond the scope of the present paper. For another interpretation of Fine's view, see Loss (2017) and Simon (2018).

12 Someone may complain that the distinction between (absolute) constitution and (irreducibly relative) obtainment is a piece of ideology, which only the fragmentalist have to endorse. This is true, but notice that the distinction does not come with acceptance of two distinct categories of properties or facts. Rather, it is a distinction between two ways facts relate to reality in the fragmentalist's picture. Thus, it may be a cost in terms of overall complexity, but not in terms of metaphysical burden, as accepting brute facts about the privileged future history is. For a detailed analysis of how to understand the notion of obtainment in a fragmentalist framework, see Iaquinto (2019, forthcoming).

13 The idea of recurring to an official idiom to avoid commitment to facts is also in Fine (2005). In this and the next sessions, we will sketch some rules and truth conditions. In the Appendix we give a more detailed exposition of the model theory. Thanks to anonymous referee for asking us to be more explicit on this aspect of our proposal. 
states that $p$. Given the fragmented nature of reality at the level of constitution, nothing about the relations between the various facts that constitute reality can be said by recurring to $L^{0}$ alone. For instance if Socrates sits, we can state in the official idiom:

(1) Socrates is sitting

and if he stands a moment after, we can state:

(2) Socrates is standing

but from (1) and (2), we cannot infer that Socrates is both sitting and standing. And in general the inferential rule of conjunction introduction (adjunction) will not hold $(\phi, \psi \not \models \phi \wedge \psi){ }^{14}$

However, if Socrates talks while he sits, then we can say that he is sitting and talking. In order to articulate this idea, we will resort to a richer language $L$, which contains not only (the fragment $L^{0}$ of) the language of tensed propositional calculus, but also operators that allow us to state what is the case relative to a fragment. The idea is to index with a number each fragment,

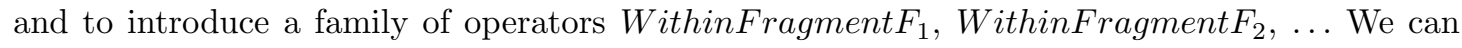
thus explicitly state not only what facts constitute reality (absolutely speaking), but also which facts obtain relative to specific fragments. The official idiom of Flow Fragmentalism will contain not only statements such as (1) and (2) but also such as:

(3) WithinFragment $F_{n}$ (Socrates is sitting)

(4) WithinFragment $F_{m}$ (Socrates is standing)

and

(5) WithinFragment $F_{n}$ (Socrates is sitting and talking)

Within the scope of such operators, the ordinary rules of sentential calculus holds. For instance, we will have the corresponding "uniformly prefixed" version of adjunction.

WithinFragment $F_{x} \phi$, WithinFragment $F_{x} \psi \vDash$ WithinFragment $F_{x}(\phi \wedge \psi)$

Besides, since if a fact obtains relative to a fragment, then it constitutes reality, we can thus safely move from a prefixed sentence to the sentence within its scope.

WithinFragment $F_{x} \phi \vDash \phi$

\footnotetext{
${ }^{14}$ This is a feature also of Loss (2017)'s interpretation of fragmentalism. Lipman (2015) maintains adjunction for conjunction, but not for co-obtainment (which can be seen as a second, non-standard, form of conjunction), for which also the converse rule of simplification does not hold (that is, from the fact that $\phi$ co-obtains with $\psi$, we cannot infer that $\phi$.). See Appendix.
} 
Therefore, while from (3) and (4) we cannot infer that Socrates is both sitting and standing (adjunction does not hold unrestrictedly), from (5) we can infer that he is both sitting and talking (if a fact obtains relatively to a fragment, it absolutely constitutes reality).

It is important here to stress that Flow Fragmentalism is a form of metaphysical pluralism, in which there are two equally fundamental ways to describe reality: the absolute constitution talk, and the fragment-relative obtainment talk. This is a crucial difference between it and other forms of non-standard tense realism. Unlike External Relativism, which vindicates Neutrality by endorsing one fundamental level of irreducible temporal perspectives, Flow Fragmentalism requires both expressive resources. And unlike other forms of fragmentalism (such as Lipman's or Loss'), Flow Fragmentalism requires a way to explicitly state what facts obtain within each fragment.

\section{Flow Fragmentalism, Present Existence, and the Flow of Time}

The distinction between which facts (absolutely) constitute reality (that is, un-prefixed sentences in the official idiom) and which facts (within a fragment) obtain (that is, prefixed sentences of the official idiom) also holds for facts about existence. Just as standard forms of tense realism are compatible with different ontologies, so are non-standard ones. Standard tense realists either accept the claim that only present things exist (i.e., they endorse standard presentism), or they deny that claim, thereby endorsing an eternalist ontology (i.e., a "moving spotlight view"), or an ontology encompassing the present and the past (i.e., a "growing block view"). Non-standard tense realists can differ from each other with respect to the facts about existence that they take to obtain within each fragment. In particular, Flow Fragmentalism encompasses the view that within each fragment, a presentist ontology holds. Thus, the minimal core of it can be articulated in the two following claims:

Ontic Flow Fragmentalism ${ }_{M C} \quad$ Within each fragment, only the present entities exist.

Factive Flow Fragmentalism ${ }_{M C} \quad$ Within each fragment, only presently obtaining facts obtain.

One may object that Flow Fragmentalism is by no means able to vindicate the idea that, in a sense, only the present is real, since facts about the existence of future and past entities do compose reality. But in so far as Flow Fragmentalism is a non-standard form of tense realism, this objection is unfair. The ontology to which a tense realist is committed depends on the 
(tensed) facts about existence that she accepts, i.e., that she accepts as obtaining. Now, while in the standard framework obtainment is absolute and for a presentist facts about existence change as time goes by (e.g., the domain of the standard presentist once contained Julius Caesar, but no longer does so), in the fragmentalist framework obtainment is irreducibly relative - hence reality does not cohere with respect to what exists, although the domain of each fragment will contain only what is present 15

Now, can Flow Fragmentalism secure bivalence for future contingents more easily than presentism? An obvious strategy to achieve this is to order the fragments in a sequence, which could play the role of a temporal succession of instants ordered by an earlier-later relation. Through such a "pseudo B-relation" $<_{p s}$, the flow fragmentalist can provide bivalent truth-conditions for future contingents in the familiar way. Imagine that two fragments $F_{1}$ and $F_{2}$ are such that within $F_{1}$ all facts that obtain at a certain instant $t_{1}$ obtain, and within $F_{2}$ all facts that obtain at a certain instant $t_{2}$ obtain. We can stipulate that $F_{1}<_{p s} F_{2}$ if and only if $t_{1}<t_{2}$. By ordering the fragments we can put them to use as instants in a standard semantics for tenses, and give fragment-relative truth-conditions for the sentences of $L$. In particular, the truth-conditions for the tense operator "WILL" and for the "WithinFragmentF $F_{x}$ operators are the following.

$[W I L L \phi]^{F_{n}}=\mathrm{T}$ if and only if there is a fragment $F_{m}$ such that (i) $F_{n}<_{p s} F_{m}$, and (ii) $[\phi]^{F_{m}}=\mathrm{T}$

$\left[\text { WithinFragment } F_{x} \phi\right]^{F_{n}}=\mathrm{T}$ if and only $[\phi]^{F_{x}}=\mathrm{T}^{16}$

Although we maintain that this idea is roughly on the right track, we see at least three problems that it must face. Firstly, $<_{p s}$ is not a temporal sequence, since it holds between fragments and not between instants. Fragments are collections of presently obtaining facts, and therefore no fragment comes before or after another. Even worse, there are literally no facts "connecting" them, since facts obtain only within fragments, and there is no "super-fragment"

15 One may have the worry that Flow Fragmentalism entails some form of commitment to non-existing objects in order to avoid contradictory talk, but this is not so. While present-tensed facts about the first child born in the next century constitute reality as much as the present-tensed fact that she or he does not (yet) exist, it is not the case that she has a certain property and she does not exist, since facts about her non existence never obtain in the same fragments where facts about her having certain (present-tensed) properties obtain.

${ }^{16}$ As we should expect, the truth value of prefixed sentences does not vary across fragments. The presence of WithinFragment $F_{x}$ operators makes the logic for $L$ described here similar to a tensed hybrid logic (cf. Blackburn 2006). 
encompassing them all within which facts concerning $<_{p s}$ can obtain. If so, one may wonder how such a view would vindicate - as tense realism in general aspires to do - the reality of the flow of time 17

Secondly, and relatedly, the flow fragmentalist seems to accept explicit quantification over fragments: "WILL $\phi$ " is true in $F_{n}$ if and only if there is a fragment $F_{m}$ such that $F_{n}<_{p s} F_{m}$, and within $F_{m}$ the fact that $\phi$ obtains. But if fragments can be quantified over, and they can play the role that instants play in standard eternalist B-theory, where exactly is the distinction between the two positions? The whole picture of a presentist metaphysics starts to fade 18

Thirdly, even if the two former difficulties can be overcome, bivalence for future contingents would be secured only if we can retrive from $<_{p s}$ a linear order. But why should that be so? After all, within each fragment the present is the only reality, and the only future facts we find are future facts in the weak sense, i.e., future-tensed facts presently obtaining. If we expand to the non-standard case the picture that we discussed at the beginning, of a tree-like structure encompassing nomological future alternatives, those facts will be about a branching, rather linear temporal topology. More precisely, in Flow Fragmentalism, future facts obtain not only relative to fragments, but also relative to nomological alternatives - viz. histories. And if this is the case within each fragment, then the relation $<_{p s}$ should be equally branching towards one of its sides. If so, resorting to it clearly cannot solve the problem of bivalence. What to do, then? In this section and in the next one, we deal with the first two difficulties, and in section 5 we will deal with the third one.

Whilst the second problem points at how to distinguish Flow Fragmentalism from the "block view", i.e. standard B-theoretic eternalism, the first one can be seen as a challenge to distinguish it from "the spotlight view", i.e. A-theoretic eternalism, in particular from its "super-time" version. As Brad Skow argues in a series of related articles on the spotlight view, we can articulate the idea that the present (or the NOW, in his terminology) moves from one instant to the next one in the temporal series, by resorting to a further dimension - viz. super-time - in which this movement takes place. Points in super-time are ordered by a relation that "mimics"

17 Leininger (2015) argues that presentism does not vindicate the passage of time (contrary to what is ordinarily held, but see Fine 2005: 286-288), because to do so requires to acknowledge the existence of the past. Although a full discussion of Leininger's argument lies behind the scope of the present paper, we believe the same objection cannot be raised against Flow fragmentalism.

18 Tallant (2013) raises a similar complaint for non-standard forms of presentism in general. One could also notice the similarity between the kind of truth-condition suggested in the text and customary tenseless accounts of tensed meaning; see, for instance, Dyke (2003). 
the linear topology and metric of B-series of instants. Thus, from the perspective of a point of super-time $T_{n}$, an instant $t_{n}$ is present, and all those coming before it (all $t_{x}$ such that $t_{x}<t_{n}$ ) are past, and all those coming after it (all $t_{x}$ such that $t_{n}<t_{x}$ ) are future. This allows us to provide an account of the flow of time as the movement of the NOW through the temporal series 19

So with supertime we can make sense of the NOW's motion: for the NOW to move is to be located at different times relative to different points of supertime. (Skow 2012:

It should be quite clear that the super-time construal of the spotlight view and non-standard tense realism bear similarities. Points of super-time closely resemble fragments (or perspectives). As with fragmentalism, facts obtain within fragments (and as with external relativism, reality is constituted by tensed facts relatively to perspectives), in the super-time story from the perspective of different points of super-time, different instants are past/present/future, and hence (we can assume) certain tensed facts rather than others obtain. Yet, there is a crucial difference between the two views.

This difference has two aspects: Skow's super-time is a metaphor ${ }^{20}$ to explain the standard form of eternalist tense realism. Fragments and perspectives are not meant to be metaphors, but to be fundamental ingredients of a metaphysical picture. But what is super-time a metaphor of? The spot-lighter has to be careful not to collapse the series of super-time points with the actual B-series of instants, on pain of collapsing her position into a form of B-theory in disguise. Thus, it cannot be a metaphor of tenseless facts concerning which instants are past, present or future relative to each other. But she must also be careful not to duplicate time in her picture, by introducing a further actual temporal series, in which the NOW can "flow". The "third way" is to construe super-time as a metaphor of irreducible tensed facts, expressed by primitive tense operators.

Talk of the NOW's motion is to be understood using primitive tense operators [...].

"The NOW is moving into the future" means (roughly) "The NOW is located at $t$,

\footnotetext{
19 Note that in his Skow (2015), though, he explores non-standard versions of tense realism and defends a "block universe" view.

20 Skow's super-time is not "hyper-time", viz. a second dimension of time, as the one sometimes discussed in the framework of time travel scenarios (see Meiland 1974 and van Inwagen 2010). Rather, it bears similarity to Schleisinger (1991)'s modal notion of "meta-time".
} 
and it will be the case that the NOW is located at a time later than $t^{\prime \prime}$. (Skow 2012:

That is why the metaphor is about a standard form of tense realism. This is a crucial aspect of the view: in the standard picture, one time is present simpliciter, and not relative to perspectives or fragments. The movement of the NOW along the super-time series is thus a metaphor for the changes in which facts are absolutely present. As Pooley rightly notes in discussing Skow's view, the problem is that there are two times in this picture: there is the A-theoretic super-time, understood in primitively tensed terms, and there is the B-theoretic time of the temporal series on which the spotlight shines and moves ${ }^{21}$ However, Flow Fragmentalism is different and it has to stand no such charge. As a non-standard form of tense realism, it accepts Neutrality and hence absolute constitution, but relativizes obtainment. Within each fragment, only one instant $t$ is present, and all past-, present-, future-tensed facts obtain at present. But all fragments are on a par, and no one corresponds to what time is present simpliciter. In the fragmentalist picture there is no movement of the absolute NOW along the series of fragments, and hence there is no super-time. Even if constitution is absolute, it reflects the inherent dynamism of the irreducibly tensed facts that obtain relative to the various fragments. Yet, if we are right, the fragments are ordered in a pseudo B-series. Thus, it seems that the fragmentalist has a two-time problem, all in all. It is that while in the spotlight view super-time is A-theoretic and normal time is B-theoretic, in the fragmentalist picture "super time" (i.e. the pseudo B-series of fragments) is B-theoretic, while ordinary time (i.e. time within each fragment) is A-theoretic.

But this distinction makes all the difference, because the flow fragmentalist - unlike the supertime spot-lighter - can avoid the two-time objection by taking the ordering of fragments by $<_{p s}$ to be a fiction. Indeed, that is precisely how we propose to solve the first predicament: $<_{p s}$ is not a temporal series, but a fiction - a fiction that does not lead to a two-time problem because, in contrast to super time, it does not encode an A-theoretic dimension built on top of a B-theoretic block universe. The reality under the fiction, as it were, is that constitution and obtainment are so arranged that if a future tensed fact (the fact that Socrates will stand, say) obtains within a fragment $F$, then its present tensed version (viz., the fact that Socrates is standing) obtains in a fragment that comes after $F$ in the series. In other terms, the fictional aspect of the pseudo B-relation $<_{p s}$ does not reside in its being a series, but rather in its being pseudo temporal: it

21 Already Williams (1951) points out a similar problem for the view that he attributes to McTaggart. Pooley (2013) dislikes the duplication of time because of the epistemic problems it gives rise to (see Braddon-Mitchell 2004). 
mimics the temporal nature of a relation between instants or events, by encoding the mutual behaviour of constitution and obtainment in the Flow Fragmentalist picture.

Thanks to the ordering of the pseudo-relation $<_{p s}$, we can move from the minimal core of flow fragmentalism to a more articulated version, as follows.

Ontic Flow Fragmentalism:
(a) Within each fragment $F$, only present entities exist.
(b) Within some fragments $F_{x}$ such that $F_{x}<_{p s} F$, past entities exist.
(c) Within some fragments $F_{x}$ such that $F<_{p s} F_{x}$, future entities exist.

Factive Flow Fragmentalism: (a) Within each fragment $F$, only presently obtaining facts obtain. Call the class of all presently obtaining facts in $F, P_{F}$.

(b) Within some fragments $F_{x}$ such that $F_{x}<_{p s} F$, present-tensed versions of the past-tensed facts in $P_{F}$ obtain.

(c) Within some fragments $F_{x}$ such that $F<_{p s} F_{x}$, present-tensed versions of the future-tensed facts in $P_{F}$ obtain.

Through the two fundamental notions of absolute constitution and relative obtainment, Flow Fragmentalism articulates the idea of an inherent dynamism of the tensed facts along the temporal dimension. From the point of view of constitution, the flow is an incoherent amalgamation of incompatible tensed facts, but at the level of obtainment, the flow is a coherent order of successively obtaining tensed facts ${ }^{22}$

Lipman (2018) argues that any form of fragmentalism that takes tense operators as primitive is not in a position to capture the idea of a genuine passage. The reason is that if presenttensed facts alone cannot explain the passage from one moment to another, neither they can together with past- and future-tensed facts. After all, we are just considering more presently obtaining facts. If Lipman is right, our position may be liable to the same criticism, given that Flow Fragmentalism purports to capture the passage of time in terms of tensed facts, and the distinction between constitution and obtainment. However, Lipman here is combining two (legitimate) criticisms in a way that leads him to a (undue) generalisation. He is right in claiming that if tenses are understood merely as indexical and perspectival tools of representation, they cannot capture genuine passage. In order to capture it, we need to introduce a primitive notion 23

And he is also right in pointing out that passage requires taking into account more that one moment of time: adding more present content to the present alone won't give us passage.

\footnotetext{
${ }^{22}$ For a more detailed elaboration of how Flow Fragmentalism captures the passage of time see Torrengo and Iaquinto 2019.

${ }^{23}$ As extensively argued in Torrengo 2018.
} 
However, those two tenets does not force us to endorse a view-such as Lipman's - in which a primitive dynamic ingredient is bestowed on the relation between facts, considered in an atemporal way. According to Flow Fragmentalism, tenses are inherently dynamic. As in the standard, Priorean picture, they are not mere indexical instruments ${ }^{24}$ they reflect the idea that if now reality is such that $p$ will be (was) the case, then reality will be (was) such that $p$. Yet, as distinctive of non-standard forms of tense realism, according to Flow Fragmentalism, the genuine form of change expressed by tenses requires that no single time is privileged. So, the difference between Lipman's account of passage and our is where to locate the primitive notion of passage: whether within each fragment, or in some fundamental relation between them. Even if Lipman's alternative is certainly viable, it strikes us somewhat against the spirit of fragmentalism. Unlike external relativism, fragmentalism does admit of an absolute way to speak of reality, yet the obtainment of a fundamental relation between distinct fragments seems to require some sort of bridging fragment, or at any rate some way to consider what happens in distinct fragments at once 25

In flow fragmentalism, there are not facts connecting distinct fragments, but only the fictional ordering $<_{p s}$ and the two equally fundamental ways of absolute constitution and relative obtainment, which allow us to consider all instants on a par, while endowing them with contradictory contents. One may wonder, whether the opposite worry can arise though. How are we to recover the ordering of $<_{p s}$, if reality is fragmented? It seems that we are never in a position to recover at once all the elements that we need to construct such series; there is no über-fragment in which facts concerning $<_{p s}$ can obtain. Pooley notices an analogous problem with respect to the external relativist account of the passage of time in terms of variation through different temporal perspectives:

Does this variation with temporal perspective provide us with a sense in which the non-standard view vindicates the passage of time? There is an apparent problem with the suggestion that it does. The variation is not itself a fact about how reality

\footnotetext{
${ }^{24} \mathrm{~A}$ diminished notion of tense, in which they are merely perspectival and indexical representational tools is, of course, also coherent (and it is possibly a more general one, applicable to anything that can be understood as a "dimension" of reality. See Torrengo 2011). We are just claiming that it is not the notion employed in Flow Fragmentalism.

${ }^{25}$ Notice also that the idea of considering a cross-temporal relation as primitively dynamic has been explored also within B-theoretic framework. See, on this, Maudlin 2007 (who Lipman quotes), but also Deng 2010 and Leininger 2018.
} 
is. Our model of the view includes such variation but [...] features of the model that transcend what is true from each temporal perspective do not correspond to perspective-independent facts about reality. There are meant to be no such facts.

(Pooley 2013: 336)

This is correct also for Flow Fragmentalism: the pseudo B-series is not grounded in facts connecting the different fragments, and cannot be, since there are no such facts. But this is how it should be. The story about $<_{p s}$ is a fiction that is not grounded in such alleged facts. There is no temporal relation linking various instants or sum of events, but only an order that mimics the temporal one. Nonetheless, the fiction is justified by the very metaphysical hypotheses about temporal reality that constitute the core of Flow Fragmentalism. By facing the second problem (how to differentiate fragmentalism-cum- $<_{p s}$ from B-theoretic eternalism) and the third one (how to recover bivalence if $<_{p s}$ is a tree-like order), we will also clarify how the fiction is to be conceived.

\section{Overlap and Branching Ordering}

The dangerous proximity between the position we advance and standard B-theory is given by the fact that we proposed to provide bivalent truth-conditions for future contingents by quantifying over fragments ordered by $<_{p s}$. It should be clear from what we said at the end of the previous section that this quantification is to be understood as part of the fiction. We have seen that the reality behind this fiction is the fact that there is an order among the fragments, even if no temporal relation holds between them. But what orders the fragment, then, if not a temporal relation between them? A hint comes from Fine himself, when he spells out the account of the flow of time in the fragmentalist picture: "Any fact is plausibly taken to belong to a 'fragment' or maximally coherent collection of facts; and so reality will divide up into a number of different but possibly overlapping fragments" (2005: 281, italics ours). Roughly speaking, two fragments are said to be partially overlapping if they share some tensed facts, such as the fact that there were dinosaurs. Intuitively, the fragmentalist can hold that, since a tensed fact of this kind is "temporal", the relation of overlap between these two fragments is sufficient to order them in a (pseudo) temporal succession. Tallant (2013) contended that

the trouble with such a proposal, aside from it being extremely controversial, is that these facts are insufficiently refined to act as suitable ground for true propositions 
about the past (and future) and when they are replaced with facts that are suitable, we find that the distinct fragments of reality will no longer overlap. (Tallant 2013: 293 , italics in the original)

As an example, Tallant proposes to consider a true proposition like 'Jonathan was hungry five minutes ago'. Its truth - he underlines - cannot be adequately grounded by the tensed fact Jonathan was hungry, but rather by the more precise tensed fact that Jonathan was hungry five minutes ago. To put it another way, the truth of 'Jonathan was hungry five minutes ago' cannot supervene on Jonathan's having been hungry, but must instead supervene on the more specific Jonathan's having been hungry five minutes ago. But it is easy to see that this more specific tensed fact cannot overlap with the fragment that represents how things will be in another minute, "for, in another minute, the tensed fact that we will require is not Jonathan's having been hungry five minutes ago, but Jonathan's having been hungry six minutes ago" (p. 294).

Nothing prevents us - Tallant concludes - from thinking of the fragments as constituted only by more specific tensed facts of this kind. But then it is hard to make sense of the claim that different fragments can overlap. Hence, the fragmentalist cannot explain how to order her fragments in a temporal sequence.

We think that Tallant's reply can be overcome. We are willing to admit that there are many propositions whose truth supervenes on "more specific" tensed facts, such as the fact that Jonathan was hungry five minutes ago. Consequently, we admit that we are required to think of fragments as constituted by such metric tensed facts. However, we disagree about whether this is sufficient for claiming that fragments cannot overlap. To be clear about the point of our reply, consider a fragment $F$ containing the tensed fact that dinosaurs became extinct at least 65 million years ago. Given that tensed fact, in $F$ the proposition 'Dinosaurs became extinct at least 65 million years ago' is obviously true. Note that if in $F$ this proposition is true, then in $F$ the proposition 'Dinosaurs became extinct at least 64 million years ago' is also true, since the former entails the latter. It follows that $F$ must also contain the tensed fact that dinosaurs became extinct at least 64 million years ago. Now, nothing prevents the fact that dinosaurs became extinct at least 64 million years ago from obtaining in another fragment - call it $F_{n}$. But then $F$ and $F_{n}$ share at least one tensed fact, namely the fact that dinosaurs became extinct at least 64 million years ago. In other terms, $F$ and $F_{n}$ are partially overlapping.

We have no reason to exclude tensed facts like dinosaurs became extinct at least 65 million years ago from the inventory of what Tallant calls "more precise" tensed facts. On the contrary, note that the former can be thought of as partially supervening on the latter. More precisely, 
they supervene on a combination of "at least" facts and "at most" facts (indeed, they can be defined in those terms, see the Appendix.)

As we have underlined in the previous section, since each fragment contains no more than presently existing things, every past/future fact that we find in a given fragment is a past/future fact in the weak sense: a past-future-tensed fact presently obtaining. The same goes for the "at least" tensed facts such as the ones that we just took into consideration: they are nonpresent weak facts and thus kosher for the presentist. The pseudo B-series of fragment can then be reconstructed out of the overlap of "at-least" facts among fragments. Of course, we are spelling out a fiction here: there are no facts about the overlap of fragments, because those would obtain only within an incoherent "über-fragment". And yet our model encompasses overlapping fragments. This is what the very hypotheses that fragments are constituted by "at least" facts, among other facts, let us conclude. Even more interestingly for our purposes, an order can also be reconstructed in the case within each fragment the tensed facts are about a branching temporal succession and the relation $<_{p s}$ is branching towards one of its sides. But how exactly is the relation of overlap sufficient for ordering the fragments along such a branching succession? Let us turn to a slightly more regimented framework.

We introduce the sentential operator $A T L E A S T_{-n}$, to be read informally as 'at least $n$ million years ago' 26 Given a present-tensed proposition such as dinosaurs become extinct, $A T L E A S T_{-n}$ (dinosaurs become extinct) is to be read as the past-tensed proposition that dinosaurs became extinct at least $n$ million years ago. Now, consider for example a fragment, $F_{0}$, containing the tensed fact that dinosaurs became extinct at least 65 million years ago, that is, a fragment in which $A T L E A S T_{-65}$ (dinosaurs become extinct) is true. Furthermore, while $F_{0}$ contains the tensed facts that dinosaurs became extinct at least 64 million years ago, that dinosaurs became extinct at least 63 million years ago, and so on and so forth, it does not contain the tensed fact that dinosaurs became extinct at least 66 million years ago. Now, we can order the fragments to be placed in the trunk whose upper bound is $F_{0}$ (see Fig. 1) by analysing how they overlap with $F_{0}$.

More precisely, given a fragment $F_{n}$, it will be part of the trunk if and only if it does not contain the tensed fact that dinosaurs became extinct at least 65 million years ago. Conversely, it will be located in one of the branches if and only if it contains the tensed fact that dinosaurs became extinct at least 65 million years ago. Analysing the overlap relation also allows us to

26 Or any other unit of time, such as days or seconds. Here we are taking into account the discrete case; the case of a dense time requires further complications that fall beyond the scope of this paper. 


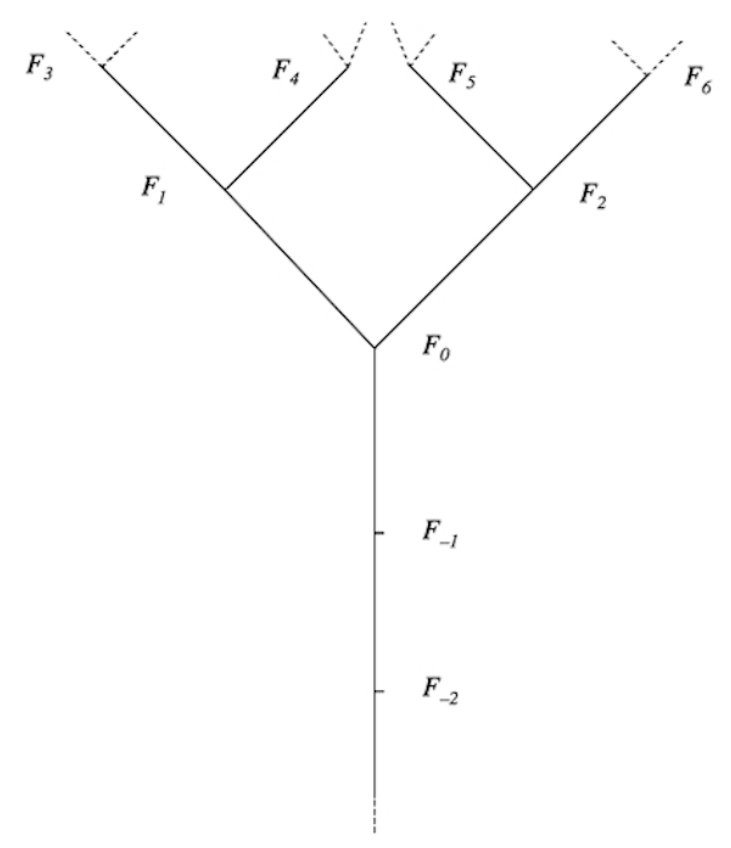

Figure 1: A branching succession of fragments with respect to $F_{0}$

determine the order in which the fragments are disposed along the trunk. Take for example two fragments $F_{-1}$ and $F_{-2}$. Suppose that $F_{-2}$ contains the tensed fact that dinosaurs became extinct at least 63 million years ago, while $F_{-1}$ also contains the tensed fact that dinosaurs became extinct at least 64 million years ago. In other words, there is at least one tensed fact obtaining in $F_{0}$ that also obtains in $F_{-1}$, but not in $F_{-2}$. In this case, $F_{-1}$ will be closer to the upper bound than $F_{-2}$ (in more formal terms, it holds that $F_{-2}<_{p s} F_{-1}$ ). To synthesise in a motto: the larger the overlap, the smaller the distance to the upper bound. This would suffice to order completely the fragment in the case of linear time (i.e., if, within each fragment, time is linear). But if the future-tensed facts within each fragment are about a branching structure, as we are assuming, we need some further refinement.

In order to calculate the distance (from the upper bound of the trunk) of the fragments that are disposed along the branches we can adopt the previous strategy, but calculating their distance to the upper bound of the trunk may no longer be sufficient for pinpointing their location. Consider Figure 1 and remember that future tensed facts obtain only relative (to fragments and) to histories ${ }^{27}$ If within $F_{0}$, relative to both history $h_{1}$ and $h_{3}$, it will be the case that dinosaurs

27 To keep things simple, we assume that $F_{1}$ and $F_{2}$ are the only "future" fragments directly connected to $F_{0}$. 
become extinct at least 66 million years ago and it will not be the case that dinosaurs become extinct at least 67 million years ago, then in the fiction there will be two fragments, $F_{1}$ and $F_{2}$ say, that contain the tensed fact that dinosaurs became extinct at least 66 million years ago (that is, Within $F_{1}$ and $F_{2}$, dinosaurs became extinct at least 66 million years ago), while they do not contain the tensed fact that dinosaurs became extinct at least 67 million years ago (that is, Within $F_{1}$ and $F_{2}$, it is not the case that dinosaurs became extinct at least 67 million years ago). It follows that they are at the same distance to $F_{0}$. This is sufficient for determining their position (in this case, the motto will be: the smaller the overlap, the larger the distance to the upper bound), but only because they are directly connected to the upper bound $F_{0}$. But what about the higher branches? Consider $F_{3}$ and $F_{5}$. Even though we are told that they are disposed to the same distance to $F_{0}$ (assume both that they contain the tensed fact that dinosaurs became extinct at least 67 million years ago and that they do not contain the tensed fact that dinosaurs became extinct at least 68 million years ago), we cannot determine either whether $F_{3}$ is connected to $F_{1}$ or to $F_{2}$ or whether $F_{5}$ is connected to $F_{1}$ or to $F_{2}$. To this end, we need a slightly more refined method. Our proposal is the following. Assume that within fragment $F_{0}$ relative to $h_{1}$ humans will become extinct in one million year, whereas relative to $h_{3}$ they won't. Then, it is true in $F_{1}$ and false in $F_{2}$ that humans are extinct. It follows that in every fragment connected to $F_{1}$ it will be true that $A T L E A S T_{-1}$ (human becomes extinct), while in every fragment connected to $F_{2}$ this proposition will be false. Hence, $F_{3}$ will be connected to $F_{1}$ if and only if it contains the tensed fact that human beings became extinct at least 1 million years ago, while $F_{5}$ will be connected to $F_{1}$ if and only if it does not contain this fact. Following this strategy, we can order the fragments in alternative histories, more precisely, pseudo-histories (given that the fragments are not ordered by the earlier-later relation, but by $<_{p s}$ ), and group them in a branching partial order.

The fiction of the branching pseudo B-relation gives us what we need to order the fragments in a way that mimics the temporal succession within each fragment, which is the reality behind the fiction. The difference with respect to the B-theory is therefore profound. According the the B-theorist, reality is constituted by all facts that obtain at all times in a coherent whole (since those facts are tenseless). According to the flow fragmentalist, there is no such coherent whole, and the order of the fragments is fictionally reconstructed from the information about the overlap between fragments which we can recover from the tensed facts that presently obtain within a given fragment. Unlike the fiction of super-time, such a fiction is B-theoretic rather than

Analogously, we assume that in the "future" of both $F_{1}$ and $F_{2}$ there are no more than two fragments. 
A-theoretic, but it does not surreptitiously reintroduce tenseless elements at the fundamental level. The pseudo B-relation is neither an irreducible tenseless relation nor a non-fundamental relation that can be reduced to tensed facts. There are no facts about $<_{p s}$ on which the fiction is grounded. The reality behind the fiction is the collection of tensed facts that obtain within one fragment relative to histories. The hypothesis that reality is as the fragmentalist says i.e. a fragmented whole of coherent collections of tensed facts - licenses the fiction of a series of partially overlapping fragments which reflects the temporal branching structure that we find within each fragment 28

\section{The Invisible Thin Red Line}

As we have just stressed, $<_{p s}$ is a partial order, such that it is linear towards one of its sides, but non-linear towards the other. If so, the fiction of a tree-like ordering of fragment is useless for providing bivalent truth-conditions for future contingents. Within each fragment time branches, and no future-tensed facts presently obtaining are privileged in any metaphysical sense. As we have pointed out in the introduction, what we need is a thin red line that singles out the actual course of events among all nomological possible alternatives. That is, we need a way to express in the fiction - which fragments contain facts that constitute reality. We could, of course, insert a thin red line within each fragment, just as the presentist can insert brute facts about which one of the possible future histories will be the actual one. For instance, within each fragment there may be matter of facts about which history is the actual one, and in the corresponding fiction about fragments ordered by $<_{p s}$ one of the histories would then be the one we can label TRL. In that way the fiction itself will contain a thin red line and bivalent truth-conditions can be given to future contingents.

But such a maneuver would condemn any account of how to single out a thin red line among

\footnotetext{
${ }^{28} \mathrm{An}$ anonymous referee has pointed out that also Lipman's primitive sentential connective passes into can be put to use to order the fragment. As for Lipman's idea of modelling the passage of time in terms of a relation between facts obtaining in different fragments (expressed by the sentential connective), we believe that our strategy, based on the fragments' overlap, is more faithful to the spirit of fragmentalism. In Lipman's model theory there is a total order relation $O$ between (points informally representing) fragments, and the semantic for passes into (and expressions in general) is given in relation to ordered couples $<t_{n}, t_{m}>$ thereof. Even if his "set-theoretic machinery is merely a heuristic tool" (p. 9), we find suspicious that sentences in the object language can be evaluated with respect to distinct fragments at once, without there being matter of facts connecting the fragments.
} 
the histories in the fiction to being circular, or at any rate grounded on the very same brute facts that we find within each fragment. As Pooley also notices, the only information we can recover within each fragment is that a single course of events will be the actual future, but not which one it will be.

While a given branching structure (absent a thin red line) does not encode a single sequence of the kind we have been considering, it does encode that the future tensed facts that hold at later and later times correspond to some such sequence. (Pooley 2013: 342$)$

Consider a very simple situation in which we ask whether it will be the case that $p$ within a fragment $F_{0}$ :

$[\text { WILLp] }]^{F_{0}}=$ ?

Assuming that $p$ is contingent, there will be two (simplifying things) future histories $h_{1}$ and $h_{2}$ such that according to $h_{1}$ it will be the case that $p$, and according to $h_{2}$ it will be the case that $\neg p$. Following the procedure described above (by resorting to the "at least" facts we find in $F_{0}$ ), we can construct a fiction in which two fragments $F_{1}$ and $F_{2}$ are both in the future of $F_{0}$ and are such that within $F_{1}$ it is the case that $p$ and within $F_{2}$ it is the case that $\neg p$.

$$
\begin{aligned}
& {[p]^{F_{1}}} \\
& {[\neg p]^{F_{2}}}
\end{aligned}
$$

In such a fiction, two sequences of fragments (two "pseudo-histories") correspond to $h_{1}$ and $h_{2}$ respectively. We can use the same labels for simplicity (see Fig. 2). Hence, while it is not settled whether within $F_{0}$ it will be the case that $p$, the fiction of $<_{p s}$ allows us to state that within $F_{0}$ relative to pseudo-history $h_{1}$ it will be the case that $p$, while relative to pseudo-history $h_{2}$ it will not:

$$
\begin{aligned}
& {[W I L L p]^{F_{0}}=\text { Ind }} \\
& {[W I L L p]^{F_{0},}, h_{1}=\mathrm{T}} \\
& {[W I L L p]^{F_{0},} h_{2}=\mathrm{F}}
\end{aligned}
$$

While the histories within a fragment $F$ are grounded in what is presently the case within $F$ and the indeterministic laws, the corresponding pseudo-histories are merely justified fictions. This is so in two senses: they are series of fragments that are not related by a temporal relation $\left(<_{p s}\right.$ is not the earlier-later relation), and (more importantly) future fragments do not exist from the 


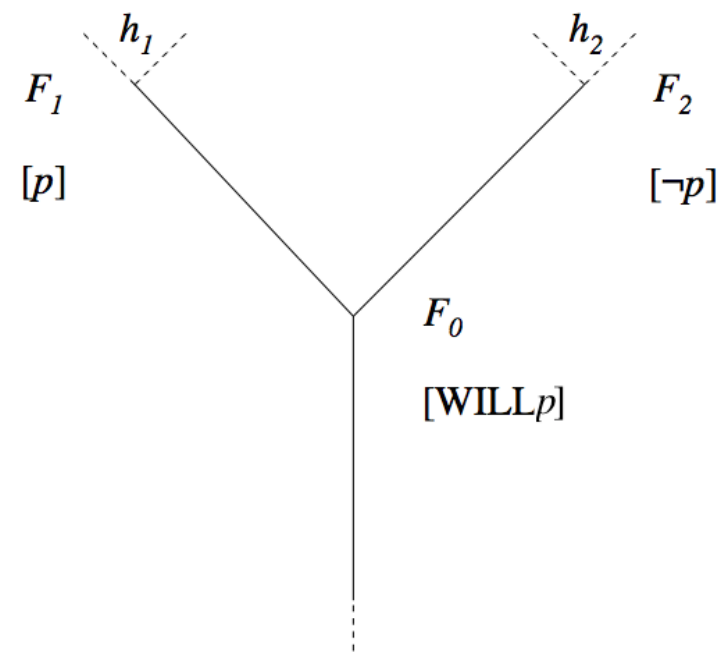

Figure 2: $h_{1}$ "passes through" $F_{1}$, while $h_{2}$ "passes through" $F_{2}$

point of view of "earlier" ones ${ }^{29}$ For instance, within fragment $F_{0}$, none (or not all, at any rate) of the facts that are supposed to obtain within $F_{1}$ and $F_{2}$ obtain. Crucially, all pseudo-histories are on a par with respect to their relation with their corresponding histories. The future tensed facts that within $F_{0}$ obtain relative to $h_{1}, h_{2}, \ldots h_{n}$ are nomological alternatives (ultimately grounded on the laws of nature and the present state of the universe), and none of them is "more real" than another.

However, the fiction that we can construct about the branching ordering of fragments is based on the information that not all fragments that constitute the different branches are part of reality. In other words, in the fiction we are postulating more fragments than there actually are. More precisely, although within each fragment non-fictional histories are on a par, and the fiction contains many sequences of fragments partially ordered by $<_{p s}$, only one of them is "out there" in the fragmented reality (see Fig. 3).

What, then, are the options for the flow fragmentalist? One is to exploit the fiction to provide supervaluationist truth-conditions for future contingents. That would save the law of excluded middle, but still jettison bivalence. Pooley seems to favour such an option, on the grounds that

\footnotetext{
${ }^{29}$ This is an aspect in which pseudo-histories resemble ersatz histories of standard presentism, as in Bourne (2006)'s and Crisp (2007)'s versions. However, while ersatz histories represent future instants, pseudo-histories are representations of different parts of a reality that is fragmented along the temporal dimension.
} 


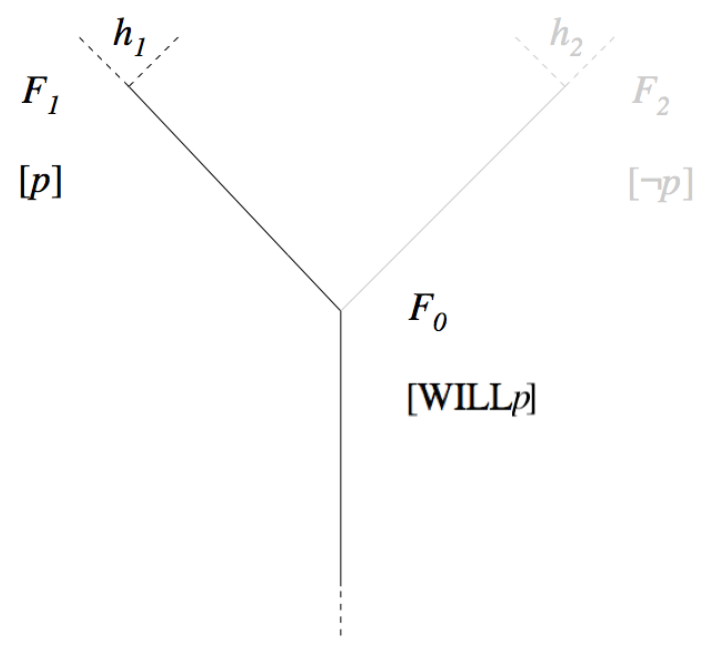

Figure 3: Reality is constituted only by facts in $h_{1}$

there is neither a "global" point of view, nor an "end of time" perspective ("the end of time is never reached" (Pooley 2013: 343)) from which we can reconstruct the information about the whole of reality. In his words:

Just as the tensed facts that hold as of some time are not reducible to tenseless facts, there is no need for them to be deducible from the tensed facts that hold as of other times. As of $t$, it is neither true nor false that there will be a sea battle at $t^{\prime}$. As of $t^{\prime}$, it is true that a sea battle is raging. [...] it might seem that this open-future version of non-standard $A$ Theory better captures the passage of time than a version in which the tensed facts as of one time can be read off from those that hold at another. In the latter case, it is hard to see what the insistence that such facts are not reducible comes to, for there is a unique representation of reality - the block universe - from which the perspectival facts can be derived. This is no longer true of the open-future model. The primordial branching structure captures only how things might turn out, not how they will turn out. (Pooley 2013: 343, italics in the original)

Also according to Flow Fragmentalism information about "future" fragments cannot be recovered from "earlier" ones. And yet there is something puzzling in the idea that the fragmentalist picture "captures only how things might turn out, not how they will turn out". Although we are barred from recovering information about the future, in the fragmentalist picture reality is 
not constituted by all facts that, in the fiction of a branching partial order of fragments, obtain within each fragment. Therefore, the very hypothesis that reality is fragmented elicits the idea that in the fiction one of the branches must be singled out as the thin red line, i.e., the one corresponding to the actual course of future events.

Of course, such a thin red line is epistemically inaccessible from within a single fragment, since within each fragment the future is not real, and we have access only to facts that obtain in the fragment we find ourselves in. If we introduce such a thin red line in the fragmentalist fiction of a pseudo B-series of fragments, then, it would be invisible. We can then label one of the pseudo-histories in the fiction the invisible thin red line [ITRL]. This situation allows us to formulate explicit bivalent truth-conditions for future contingents. Here is the clause for the WILL operator. We need first to define truth relative to a fragment $F_{n}$ that "passes through" a pseudo-history $h_{x}$.

$[W I L L \phi]^{F_{n}, h_{x}}=\mathrm{T}$ if and only if there is a fragment $F_{m}$ that passes through $h_{x}$ such that $F_{n}<_{p s} F_{m}$, and $[\phi]^{F_{m}, h_{x}}=\mathrm{T}$

Truth relative to a fragment $F_{n}$ simpliciter is truth relative to $F_{n}$ and the ITRL (for a more general formulation of those ideas see the Appendix).

$$
[\phi]^{F_{n}}=\mathrm{T} \text { if and only if }[\phi]^{F_{n}, I T R L}=\mathrm{T}
$$

It is important to stress that "invisibility" here is not to be understood in merely epistemic terms. There is literally no fact of the matter as to which of the pseudo-histories in the fiction correspond to the actual future ${ }^{30}$ However, in order to select a ITRL, we do not need an über-fragment in which facts that can ground it obtain. As with the construction of the tree of fragments, the selection itself of a ITRL among the pseudo-histories is part of the fiction. We can think of it as a "prima facie assignment" of the role of ITRL to one of the pseudo-histories, along the lines of the provisional selection of a possible future to evaluate future tensed statements in a "Ockhamist"

30 Indeed, if the ITRL were determined by facts about the whole of reality, this would be in tension with the idea that reality is not of a whole, but fragmented. Flow Fragmentalism does not entail the thesis that Loss (2019: 1253) discusses, according to which the overall structure of the fragmented reality can determine within each fragment a TRL. Thanks to an anonymous referee for having asked to clarify the difference, if any, between our proposal and that passage of Loss'. 
framework 31 With the important proviso that we have a justification for introducing such a further element in the fiction. More precisely, it is the metaphysical hypothesis that reality is made of incoherent fragments in which incompatible tensed facts obtain, together with the possibility of cooking up a story about their ordering, that justifies the introduction of a unique actual future. Since within each fragment the future branches, but ex hypothesis there is one fragmented reality with one temporal dimension, it would be unfaithful to the metaphysics that we are assuming not to postulate a ITRL in the fiction.

That is why, as distinct from presentism, adding a thin red line is no extra cost for the flow fragmentalist. In the standard picture, having a thin red line entails accepting facts about what the actual future will be within the perspective of the present time, which is the only real perspective. Such facts are either brute or come at additional costs in terms of primitive ideology. But in the fragmentalist version of the story, we are not required to accept facts about the actual future within each fragment. If fragmentalism is true, we know that an invisible thin red line can't fail to be out there, since only the fragments that form a certain sequence in the fiction are part of reality; all others are not part of reality at all. At one point, Pooley seems to be sympathetic to such an idea:

The model of the non-standard variant of the view does involve a particular sequence [a sequence of perspectives that stands for the actual future course of events]. Each element of it represents the irreducibly tensed facts that hold as of some time. This might seem to give us a more explicit representation of once open possibilities being settled by the passage of time: what is indeterminate as of $t$ is settled in such-andsuch a way as of $t^{\prime}$. But care is needed: the sequence of trees does not represent how reality is absolutely, as conceived from no particular temporal point of view. (Pooley 2013: 342 , italics in the original)

As we have seen, "care" pushes Pooley to reject the idea that the fact that "the view does involve a particular sequence" justifies the endorsement of a thin red line. That may be because Pooley uses a different version of non-standard tense realism from us - external relativism on his part, and fragmentalism on ours.

\footnotetext{
${ }^{31}$ The idea is in Thomason (1970: 270-1), who is elaborating on Prior (1967: Chap. 7). Thomason argues that understanding the selection of a history for semantic evaluation as provisional is "an unstable view" (p. 271), since it does not warrant bivalence- - but his rationale assumes that there is no justification for maintaining that there is a unique actual future.
} 
Remember that while both external relativism and fragmentalism accept Neutrality, external relativism rejects Absolutism while fragmentalism rejects Coherence, as we repeat below.

Absolutism The constitution of reality is an absolute matter, i.e. not relative to a time or other form of temporal standpoint.

Coherence Reality is not contradictory; it is not constituted by facts with incompatible content.

Therefore, the fragmentalist does not accept that the constitution of reality is irreducibly relative to fragments (or perspectives, or points in super-time, or what have you); although she does relativize what facts obtain to fragments: we are never allowed to claim that facts that we find in a different fragment from the one in which certain facts obtain also obtain ${ }^{32}$ The fragmentalist reality is not "of a whole" because as a whole it would be incoherent, but it is nonetheless constituted by all tensed facts in a absolute sense. On the other hand, in the external relativist picture, tensed facts do not constitute one reality, since they only constitute reality relative to perspectives. This makes a difference when it comes to the fiction of ordering the fragment with $<_{p s}$. In an external relativist framework, it is not only that we don't find a global perspective or a perspective as of the end of time, we do not find a reality constituted by all the facts that we find along the thin red line, indeed along any of the fictional fragments. Hence, in an external relativist framework the postulation of a thin red line would be a fiction about a further reality constituted by incompatible facts. This may be a price that someone endorsing such a version of non-standard tense realism - as Pooley in the paper we just quoted - may not be willing to accept. But in the fragmentalist version, since Coherence but not Absolutism is dropped, the postulation of such a reality is no additional cost at all; indeed, an incoherent but fragmented whole of incompatible tensed facts is the only reality that the model posits. And although the metaphysical hypotheses concerning such a reality entail that neither the branching order of the fragments nor the branch that corresponds to the actual future can even in principle be "seen" (since no facts that ground them obtain), those very hypotheses entitle us to construct a fiction about a sequence of fragments and an invisible thin red line.

\section{Appendix: the Model Theory}

Syntactic rules for $L$

\footnotetext{
32 Fine (2005: 297) claims: "In stating that a fact belongs to reality, we adopt a general perspective, but in stating that a fact obtains, we adopt the current perspective". Again, such claims do not merely reflect limitations in what we can express, but encode substantive metaphysical theses.
} 
(a) $p_{1}, p_{2}, \ldots$ are wffs (the atoms of $L$ )

(b) If $\phi$ and $\psi$ are wffs, then $\neg \phi$ and $\phi \wedge \psi$ are wffs

(c) If $\phi$ is a wff, and $x$ and $y$ are numbers, then $A T L E A S T_{x} \phi, A T M O S T_{x} \phi$, and WithinFragment $F_{\left(i_{x}, h_{y}\right)} \phi$ are wffs

The Model $\mathcal{M}$

A Model $\mathcal{M}$ is a tuple $\left\langle\mathcal{F},<_{p s}, d, I T R L, V\right\rangle$ such that:

(d) $\mathcal{F}=\left\{F_{1}, F_{2}, \ldots\right\}$ is a set of points, informally representing the fragments;

(e) $<_{p s}$ is a partial order on $\mathcal{F}$ that is linear towards the left and branching towards the right, and it is such that any two points have a common $\leq_{p s}$-ancestor, thus forming a tree structure. Pseudo-histories $h, h_{1}, \ldots$ can be defined as sets of maximal chains of $\leq_{p s}$-connected points.

(f) $d$ is a distance function that, for each pair of $\leq_{p s}$-connected points in $\mathcal{F}$, gives a positive number $x$ expressing their distance in time units (see Koymans 1990 for details). Two points $F_{x}, F_{y}$ are said to be $d$-aligned iff they lie at the same temporal distance from a point $F_{z}$ that (improperly) precedes both of them. Instants are defined as equivalence classes of $d$-aligned moments. We let $i_{0}$ be an arbitrary instant and, for any instant $i_{x}$, we write $i_{x-n}$ to indicate the instant that is $n$ time units in the past of $i_{x}$, and we write $i_{x+n}$ to indicate the instant that is $n$ time units in the future of $i_{x}$.

(g) We require that the tree be synchronised in the sense of Di Maio and Zanardo (1994: 269$273)$, so that any instant $i_{x}$ intersects each pseudo-history $h_{y}$ on precisely one point ${ }^{33}$ We write $\left(i_{x}, h_{y}\right)$ to indicate the unique point lying in the intersection of $i_{x}$ and $h_{y}$.

(h) ITRL is a selected pseudo-history, which informally represents the invisible thin red line, the sequence of fragments that constitutes the actual history of the universe.

(i) $V$ is an evaluation function that associates each atom in $L$ with a function from points in $\mathcal{F}$ to truth values in $\{T, F\}$.

The Semantics

(j) $[p]_{\mathcal{M}}^{\left(i_{x}, h_{y}\right)}=T$ iff $V(p)\left(i_{x}, h_{y}\right)=T(p$ atomic; reference to $\mathcal{M}$ omitted for brevity henceforth $)$

(k) $[\neg \phi]_{\mathcal{M}}^{\left(i_{x}, h_{y}\right)}=T$ iff $[\phi]_{\mathcal{M}}^{\left(i_{x}, h_{y}\right)} \neq T$

(l) $[\phi \wedge \psi]^{\left(i_{x}, h_{y}\right)}=T$ iff $[\phi]^{\left(i_{x}, h_{y}\right)}=T$ and $[\psi]^{\left(i_{x}, h_{y}\right)}=T$

(m) $\left[A T L E A S T_{n} \phi\right]^{\left(i_{x}, h_{y}\right)}=T$ iff for some $k$ such that $k \leqslant(x-n),[\phi]^{\left(i_{k}, h_{y}\right)}=T$

(n) $\left[A T M O S T_{n} \phi\right]^{\left(i_{x}, h_{y}\right)}=T$ iff there is a $k$ such that $(x-n) \leqslant k,[\phi]^{\left(i_{k}, h_{y}\right)}=T$

\footnotetext{
${ }^{33}$ See Spolaore and Gallina (2018: $\S 2$ ) for an example of syncronised metric tree structure.
} 
(o) $\left[\text { WithinFragment } F_{\left(i_{w}, h_{z}\right)} \phi\right]^{\left(i_{x}, h_{y}\right)}=T$ iff $[\phi]^{\left(i_{w}, h_{z}\right)}=T$

We can define the following operators:

(p.1) $T E N S E_{n} \phi={ }_{d f} . A T L E A S T_{n} \phi \wedge A T M O S T_{n} \phi$

(p.2) $W A S_{n} \phi=_{d f}$. $T E N S E_{n} \phi$, when $n$ is positive

(p.3) $W I L L_{n} \phi=_{d f}$. TENSE $E_{n} \phi$, when $n$ is negative

(p.4) $W A S \phi={ }_{d f}$. for some $n, W A S_{n} \phi$

(p.5) $W I L L \phi=_{d f}$. for some $n, W I L L_{n} \phi$

Truth at an instant is defined as follows:

(q) $[\phi]^{i_{x}}=T$ iff $[\phi]^{\left(i_{x}, I T R L\right)}=T$

Truth in $\mathcal{M}$, logical consequence, and validity

Truth in a model $\mathcal{M}$ can be defined in a non recursive way as follows.

(r) $\mathcal{M} \vDash \phi$ iff for some $i_{x},[\phi]_{\mathcal{M}}^{i_{x}}=T$

Logical consequence and validity are defined globally as follows.

(s) $\Sigma \vDash \phi$, if and only if, for any model $\mathcal{M}$, if $\mathcal{M} \vDash \Sigma$, then $\mathcal{M} \vDash \phi$

(t) $\vDash \phi$, if and only if, for any model $\mathcal{M}, \mathcal{M} \vDash \phi$

As expected, the semantic behaviour of conjunction (l) warrants that adjunction will fail in general.

(u) $\phi, \psi \not \models \phi \wedge \psi$

However, as stated in the text, the "uniformly prefixed" version of adjunction holds.

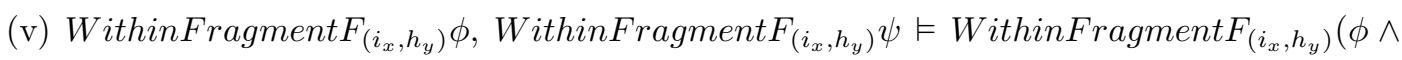
$\psi)$

Besides, for any fragment $F_{\left(i_{x}, h_{y}\right)}$ which is on the ITRL, we have that from a sentence whose main operator is WithinFragment $F_{\left(i_{x}, h_{y}\right)}$ we can infer the sentence within its scope.

(w) WithinFragment $F_{\left(i_{x}, h_{y}\right)} \phi \vDash \phi$

Note that even contradictory pairs of sentences $\phi, \neg \phi$ can both be true in a model, while there is no model where their conjunction $(\phi \wedge \neg \phi)$ is true. Given that we endorse a local notion of negation (that is, we endorse $(\mathrm{k})$ together with $(\mathrm{r})$ ), this is as it should be. If within a fragment there is not good weather in Baltimore, then the fact that there is not good weather in Baltimore constitutes reality, even if in a different fragment there is good weather in Baltimore, and thus also the fact that there is good weather in Baltimore constitutes reality. 
Our approach has two notable consequences. Firstly, we do not have to take a stance on which atomic sentences express instantiation of "positive" properties, and which ones simply express failure of instantiation thereof: it does not matter whether good weather is just lack of bad weather or the other way around. It seems to us a good result that from a metaphysic of temporal reality, no substantive commitments on the nature of properties follows. The second one is that while adjunction fails, simplification holds.

(x) $\phi \wedge \psi \vDash \phi, \psi$

This is to be expected, if the fact that $\phi \wedge \psi$ constitutes reality, then there is a fragment (on the ITRL) where both $\phi$ and $\psi$ hold, and thus both that fact that $\phi$, and the fact that $\psi$ will constitute reality ${ }^{34}$

Prof. Giuliano Torrengo Centre for Philosophy of Time, University of Milan \& Autònoma University of Barcelona

Dr. Samuele Iaquinto Centre for Philosophy of Time, University of Milan

\section{References}

Barnes, E., Cameron, R. 2009. "The Open Future: Bivalence, Determinism and Ontology". Philosophical Studies 146: 291-309

Belnap, N. et al. 2001. Facing the Future. Agents and Choices in Our Indeterminist World. Oxford: OUP

Blackburn, P. 2006. "Arthur Prior and Hybrid Logic", Synthese, 150, 3: 329-372

Bigelow, J. 1996. "Presentism and Properties". Philosophical Perspectives 10: 35-52

Borghini, A., Torrengo, G. 2012. "The Metaphysics of the Thin Red Line". In F. Correia, A. Iacona (eds.). Around the Tree. Berlin: Springer Verlag, pp. 105-125

Bourne, C. 2006. A Future for Presentism. Oxford: OUP

Braddon-Mitchell, D. 2004. "How Do We Know it is Now Now?". Analysis 64: 199-203

\footnotetext{
${ }^{34}$ Those are major differences with Lipman (2018)'s model theory. Given that he defines truth in a model (recursively) in a way that reflects a global conception of negation, the fact that $\neg \phi$ constitutes reality only if nowhere in reality it is the case. If $\neg \phi$ is the case within a fragment, but $\phi$ is the case within some other fragment, then the fact that $\phi$ will constitute reality, but not the fact that $\neg \phi$. As Lipman rightly notes, his approach requires that we decide which sentences express instantiation of "positive" properties. Moreover, given that negation in Lipman's framework behaves differently whether it is the main operator or it is embedded, both adjunction and simplification for co-obtainment fail.
} 
Brogaard, B. 2006. "Tensed Relations". Analysis 66: 194-202

Brogaard, B. 2013. "Presentism, Primitivism and Cross-temporal Relations: Lessons from Holistic Ersatzism and Dynamic Semantics". In R. Ciuni, K. Miller, G. Torrengo (eds.). New Papers on the Present: Focus on Presentism. Munich, Philosophia Verlag, pp. 253-277

Cameron, R.P. 2015. The Moving Spotlight. An Essay on Time and Ontology. Oxford: OUP

Correia, F., Rosenkranz, S. 2011. "Eternal Facts in an Ageing Universe". Australasian Journal of Philosophy 90: 307-320

Crisp, T. 2007. "Presentism and the Grounding Objection". Nô̂s 41: 90-109

Di Maio, M. C., \& Zanardo, A. (1994). "Synchronized histories in Prior-Thomason representation of branching time." In D. Gabbay \& H. Ohlbach (Eds.), Proceedings of the first international conference on temporal logic, Dordrecht, Springer: 265-282

Deng, N. 2010, "Beyond A- and B-Time? Reconsidered", Philosophia, 38, 4: 741-753

Dyke, H. 2003. "Tensed Meaning: A Tenseless Account". Journal of Philosophical Research 27: $67-83$

Fine, K. 2005. "Tense and Reality". In K. Fine. Modality and Tense. Philosophical Papers. Oxford: OUP, pp. 261-320

Fine, K. 2006. "The Reality of Tense". Synthese 150: 399-414

Iaquinto, S. 2019. "Fragmentalist Presentist Perdurantism". Philosophia 47: 693-703

Iaquinto, S. forthcoming. "Modal Fragmentalism". The Philosophical Quarterly. Online first Ingram, D. 2016a. "The Virtues of Thisness Presentism". Philosophical Studies 173: 2867-2888 Ingram, D. 2016b. "Thisnesses, Propositions, and Truth". Pacific Philosophical Quarterly. Available online from 6 December 2016. DOI: 10.1111/papq.12181

Keller, S. 2004. "Presentism and Truthmaking". In D. Zimmerman (ed.). Oxford Studies in Metaphysics, Vol. 1. Oxford: OUP, pp. 83-104.

Koymans, R. 1990. "Specifying real-time properties with metric temporal logic". Real-Time Systems, 2(4): 255-299

Le Poidevin, R. 1991. Change, Cause and Contradiction: A Defence of the Tenseless Theory of Time. London: Macmillan

Leininger, L. (2018) "Objective Becoming: In Search of A-ness", thextitAnalysis 78 (1): pp. 108-117

Leininger, L. 2015. "Presentism and the Myth of Passage". Australasian Journal of Philosophy 93: $724-739$

Lipman, M. A. 2015. “On Fine's Fragmentalism”. Philosophical Studies 172: 3119-3133 
Lipman, M. A. 2018. "A Passage Theory of Time.” In Oxford Studies in Metaphysics, 11, edited by Karen Bennett and Dean Zimmerman, 95-122. Oxford: Oxford University Press.

Loss, R. 2017. 'Fine's McTaggart: Reloaded'. Manuscrito 40: 209-239.

Loss, R. 2019. "No Ground for Doomsday." Inquiry, 62(9-10): 1136-56.

Markosian, N. 2013. "The Truth About the Past and the Future". In F. Correia, A. Iacona (eds.). Around the Tree. Berlin: Springer Verlag, pp. 127-142

McTaggart, J.M.E. 1908. "The Unreality of Time". Mind 17: 457-73. Reprinted in R. Le Poidevin, M. McBeath (eds.). 1993. The Philosophy of Time. Oxford: OUP, pp. 23-34

Merlo, G. (2013) "Specialness and egalitarianism". Thought: A Journal of Philosophy 2 (3): 248-257

Meiland, J.W. 1974. "A Two Dimensional Passage Model of Time for Time Travel". Philosophical Studies 26: 153-173

Øhrstrøm, P. 2009. "In Defense of the Thin Red Line". Humana.mente 8 - Models of Time: $17-32$

Pooley, O. 2013. "Relativity, the Open Future, and the Passage of Time". Proceedings of the Aristotelian Society 113 (Part 3): 321-363

Prior, A. 1967. Past, Present, and Future. Oxford: OUP

Schleisinger, G.N. 1991. "E Pur Si Muove". The Philosophical Quarterly 41: 427-441

Skow, B. 2009. "Relativity and the Moving Spotlight". Journal of Philosophy 106: 666-678

Skow, B. 2012. "Why Does Time Pass?". Nô̂s 46: 223-242

Skow, B. 2015. Objective Becoming. Oxford: OUP

Simon, J. 2018. "Fragmenting the wave function." In Oxford Studies in Metaphysics, Volume 11, edited by Karen Bennett and Dean Zimmerman, 123?145. Oxford: Oxford University Press.

Spolaore G. and Gallina F., 2017. "The Actual Future is Open", Erkenntnis. Online first

Tallant, J. 2013. "A Heterodox Presentism: Kit Fine's theory". In R. Ciuni, K. Miller, G. Torrengo (eds.). New Papers on the Present. Focus on Presentism. Munchen: Philosophia Verlag, pp. 281-305

Thomason, R.H. (1970). "Indeterminist time and truth value gap," Theoria, 34, 3: 264-81

Torre, S. 2011. "The Open Future". Philosophy Compass 6: 360-373

Torrengo, G. (2011) "The Modal Dimension," Humana.Mente Journal of Philosophical Studies, 19: pp.105-120

Torrengo, G. 2018. "Perspectival Tenses and Dynamic Tenses". Erkenntnis83(5): pp. 1045-1061 
Torrengo, G. and Iaquinto S. 2019. "Flow Fragmentalism". Theoria 85: 185-201

van Inwagen, P. 2010. "Changing the Past". In D. Zimmerman (ed.). Oxford Studies in Metaphysics. Vol. 5. Oxford: OUP, pp. 3-28

Williams, D.C. 1951. "The Myth of Passage". The Journal of Philosophy 48: 457-472 